\title{
Mucociliary clearance-therapeutic aids
}

\author{
Volume 8 Issue I - 2017 \\ Murray Grossan \\ American Board of Otolaryngology Head and Neck Surgery, \\ USA
}

Keywords: mucociliary clearance, cilia beat frequency, therapeutic sids, healing respiratory pathologies, healthy body

Abbreviations: MCC, mucociliary clearance; $\mathrm{CBF}$, cilia beat frequency; LRT, lower respiratory tract

\section{Introduction}

There are excellent programs detailing standards for treating Sinusitis. There detail recommended antibiotic therapy. However, as the numbers of resistant bacteria increase, it is valuable to review aids that can aid in healing respiratory infections, with or without antibiotics. For this purpose, I review here aids for Mucociliary Clearance, which can significantly aid in healing respiratory pathologies.

\section{Mucociliary clearance definition}

In the healthy body, Mucociliary Clearance (MCC) moves bacteria and dust out of the nose, to the back of the throat, down to the stomach where acid detoxifies the bacteria. ${ }^{1}$ If the MCC is slowed, then bacteria remain in place, and multiply. If MCC is slowed, then mucus accumulates, and thickens. This further slows cilia movement. If the mucus thickens, this inhabits Cilia Beat Frequency (CBF). ${ }^{2}$

Cilia are in a lower layer that is thin. The upper layer - the gel layer contains goblet cells, submucosal glands, mucin, IgA, albumin, lactoferrin and lysozyme. Normally between 1-2 liters of mucus are produced daily. Synchronous movement of the cilia moves the upper gel layer.

\section{The saccharine test of MCL}

Clinically, measuring CBF is not difficult. The Saccharine test of nasal cilia function is recognized as a useful test. Simply place a particle of Saccharine into the nose $0.5 \mathrm{~cm}$ behind the columella. Have the patient sit quietly and not sniff or sneeze. The nasal cilia will propel the saccharin particle to the back of the throat where it is swallowed and tasted. The normal person tastes this in 5-8minutes. Slow cilia are about 8-20minutes and are seen in postnasal drip and chronic sinusitis. ${ }^{3}$

Normal CBF is $12-15 \mathrm{hz}$. At this rate, a particle of saccharine placed $0.5 \mathrm{~cm}$ into the turbinate will reach the tongue and be tasted in 5-7 minutes. The CBF of the nose is thought to match that of the CBF of the lower respiratory tract (LRT). This follows the Unified Field Theory that holds that effects on the MCF effect the entire respiratory system. In one study, patients hospitalized with COPD, bronchitis, or pneumonia showed impaired nasal $\mathrm{CBF}^{4,5}$

\section{Allergy and CBF}

In Acute Allergy the cilia speed up. Mucus becomes thinner, and more copious to allow faster cilia movement and better dilution of the antigen. In the acute allergy, the saccharine particle will reach the tongue in 1-4minutes. However, as the duration of the seasonal allergy drags on, the CBF slows down. This is a reason why some patients report sinus infection after weeks of allergy symptoms. Attention to restoring cilia at this time may avoid the sinus infection. ${ }^{6}$
Correspondence: Murray Grossan American Board of Otolaryngology Head and Neck Surgery Cedars Sinai Medical Towers 863I W Third St Suite 440 East Los Angeles Ca 90048 , USA, Tel (310) 659-I006, Email drgrossan@yahoo.com

Received: August 16, 2016 | Published: July 18, 2017

\section{Post- nasal surgery}

Several studies have demonstrated slowing of the cilia for weeks after any nasal surgery. This is one reason why some doctors place patients on antibiotics following nasal surgery. Instead of antibiotics, attention to restoring nasal cilia may be preferred following nasal surgery. ${ }^{7}$

\section{Toxic products}

The number of toxins that impair cilia include: chlorine, chromium, cadmium, nickel, copper, hair spray, and wood dust. Atmospheric Products that slow MCC include O3, SO2, NO2, and NH3. However, in smog, these can form multiple compounds that are harmful.

Most toxic of all is chlorine gas. In World War One those who survived a chlorine gas attack, but inhaled chlorine, often went on to succumb to pneumonia because of poor cilia of the respiratory tract. ${ }^{8}$ $\mathrm{Smog}$ can have $\mathrm{SO} 2$ or combinations of $\mathrm{SO} 2$ and other products. The cough of smog is related to reduce cilia of the lower respiratory tract that results in the cough as a means of removing thick mucus. Many industrial solvents and aromatic products impair cilia. Volcanic gases are toxic in proportion to the $\mathrm{SO} 2$, Chlorine, $\mathrm{NO} 2$ and $\mathrm{NH} 3$ products and combinations.

Some bacterial pathogens specifically target ciliated cells for adherence, including Actinobacillus pleuropneumonia, Pseudomonas aeruginosa, Moraxella catarrhalis, Mycoplasma pneumonia, Mycoplasma hyopneumoniae and Bordatella species.

\section{Aids to CBF}

$\beta$-agonists, including salmeterol and salbutamol, raise intracellular cAMP levels and thereby increase cilia beat frequency. Consistent with this observation, inhalation of salbutamol increases mucociliary clearance in chronic bronchitis, as does theophylline and aminophylline. ${ }^{9}$

In patients with nasal polyps, CBF is not decreased. ${ }^{10}$ The high incidence of sinusitis and bronchitis among the $9 / 11$ rescuers is related to failure of MCC to return after inhalation of toxic dusts.

Cystic Fibrosis Patients are benefitted by the use of inhaled hypertonic saline and salbuanol which thins the thick mucus by osmotic pressure. 


\section{Clinical discussion}

Exposure to smoke is especially harmful to CBF of children. ${ }^{11}$ Intubation with high pressure oxygen slows CBF. Hyperbaric oxygen impairs cilia function. Various genetic conditions exist that result in asynchronous cilia beating, or other forms of cilia dyskinesia. These are characterized by multiple sinus and pulmonary infections. In Cystic Fibrosis, the outer gel layer is thickened, so that it inhibits cilia movement. Patients receiving CPAP for sleep apnea show normal $\mathrm{CBF}$ when moisture is added. ${ }^{12}$

\section{Industry}

When patients complain of odors causing nasal dryness, cough, sinus infection, or postnasal drip, the saccharine test is useful to demonstrate CBF. When new solvents or chemicals are introduced, effect on CBF are not evaluated, much less listed. When workers complain of nasal symptoms due to the chemical exposure, measuring MCC via the Saccharine test can be very useful. ${ }^{13}$

\section{Winter colds}

Winter colds are common. One factor is that nasal cilia slow down in cold air. With CBF reduced, when the person rushes out of the cold into the classroom or elevator, the bacteria that enter may remain in place and replicate, thus causing a fresh infection. Ideally, from the cold, one should sit and drink hot tea before entering the classroom. This is rarely possible: however, warming up away from infected persons can make a huge difference. In school, there is often a hallway one can stroll in first before entering the classroom, to allow cilia to recover from the cold. Breathing into the cupped hands in also an aid. $^{14}$

\section{Practical therapy}

Therapy for MCC, involves thinning the mucus and speeding the cilia. For centuries Yoga practitioners have practiced humming "ooommm." This can be effective because of the low $\mathrm{Hz}$ speed of the sound. This vibration acts to break up the gel bonds for thinner mucus. They have also promoted saline nasal irrigation which removes thick mucus to aid CBF.

Regular "ooommm", humming low toned songs can aid both upper and lower respiratory CBF. Increasing intake of warm water is an aid too. Tea, particularly green tea with lemon is effective. Tea, Green or Black, contains L-theonine an important aid to immunity. Doctors recommend chicken soup for $\mathrm{CBF}$. Chicken soup contains an amino acid called L-cysteine that is released when you make the soup. This amino acid thins mucus aiding in the healing process.

\section{Solutions for CBF}

Wilbert Boek, of Utrech, compared CBF of various nasal solutions. $\mathrm{He}$ found that that the Locke Ringer solution that included Potassium and Calcium chloride was most effective. Clinically, use of the Locke Ringer's formula for nasal/sinus irrigation may be more effective than plain normal saline. ${ }^{15}$

Prepared saline solutions marketed for irrigation usually contain preservative products such as Benzalkonium. These and similar products are known to impair CBF. When patients show poor result with saline irrigation, it is important to check for the presence of preservatives. ${ }^{16}$ Changing to a saline solution that is free of preservatives may show improvement of symptoms.

Proteolytic enzymes such as bromelain and papain are used: dissolve in the buccal pouch to thin the mucus and reduce swelling. Since these enzymes are inactivated by stomach contents, by having them melt in the buccal pouch, there is better absorption.

\section{Pulsed irrigation}

When I visited yoga master Indra Devi, she demonstrated how she and her students avoided infection. She would sniff saline rapidly in and out at a set speed and pressure. I recognized that this rapid pulsing action was ideal for stimulating nasal cilia. Hence, using a pulsed stream with the rate of pulsation geared to cilia pulse rate does aid in improving $\mathrm{CBF}$.

Devices, such as the Hydro Pulse ${ }^{\mathrm{TM}}$ can maintain a steady stream at a steady pressure. With pulsed irrigation, the thickened mucus is removed, and the cilia are rocked at a desirable pace. This results in a Bernoulli vacuum effect as it flows past the sinus ostia; this suction helps remove sinus contents. Dr. Terence Davidson, UC San Diego, recommended adding antibiotics to the pulsed irrigation.

\section{Direct sinus irrigation}

In skilled experienced hands, direct irrigation via an inserted cannula is an ideal way to drain a blocked sinus. Via the cannula, medication can be inserted too. In some patients, restoring CBF may act to drain blocked sinuses too. Even though the CT scan shows a blocked sinus, restoring $\mathrm{CBF}$ may result in an open sinus in some cases. Of interest is the work of Sisson that shows alcohol simulates ciliary motility. ${ }^{17}$

\section{Summary Grossan ${ }^{18}$}

It is convenient that various methods of improving CBF can be clinically evaluated by performing the saccharine test before and after therapy. This enables doctors to evaluate methods that work for their patients, and has speeded the development of methods of improving CBF.

For patients who exhibit reduced CBF, therapy methods include:

a. Humming including "ooommm."

b. Tea, especially green tea with lemon.

c. Increasing liquid intake

d. Chicken soup

e. Saline Irrigation

f. Pulsed irrigation using saline formulated according to Boek's Locke Ringer Formula

g. Proteolytic enzymes- dissolved in buccal pouch to thin the mucus

h. Certain spices - horseradish, garlic and wasabi.

\section{Future research}

Measuring $\mathrm{CBF}$ in the lower respiratory tract is difficult. Some studies suggest that $\mathrm{CBF}$ in the nose often reflects $\mathrm{CBF}$ in the lower respiratory tract. An important new research would be to determine if simply increasing CBF in the nose actually benefits CBF in the lower respiratory system. If research did indicate that $\mathrm{CBF}$ was improved in the lower respiratory system by improving it in the upper, this would open up a new mode of therapy for chronic bronchitis, pneumonia, COPD and many other lower respiratory conditions. I would like to urge readers to consider this research. 


\section{Funding}

No funding for this article.

\section{Conflicts of interest}

At the time of this writing, I am no longer president of Hydro Med Inc. Hydro Med Inc. was sold to Neil Med Pharmaceutical September 2016 and I remain a consultant to that company.

\section{Acknowledgments}

None.

\section{References}

1. Hens G, Hellings PW. The Nose: Gatekeeper and Trigger of Bronchial Disease. Rhinology. 2006;44(3):179-187.

2. Knowles MR, Boucher RC. Mucus clearance as a primary innate defense mechanism for mammalian airways. J Clin Invest . 2002;109(5):571-577.

3. Grossan M. The Saccharin Test of Nasal Mucociliary Function. Eye Ear Nose Throat Mon. 1975;54(11):415-417.

4. Braunstahl GJ. United Airways Concept: What Does It Teach Us about Systemic Inflammation in Airways Disease? Proc Am Thorac Soc. 2009;6(8):652-654.

5. Hens G, Vanaudenaerde BM, Bullens DM, et al. Sinonasal Pathology in Nonallergic Asthma and COPD: 'United Airway Disease' beyond the Scope of Allergy. Allergy. 2008;63(3):261-267.

6. Sleigh MA, Blake JR, Liron N. The propulsion of mucus by cilia. Am Rev Respir Dis 137(3): 726-741.

7. Braiman A, Priel Z (2008) Efficient mucociliary transport relies on efficient regulation of ciliary beating. Respir Physiol Neurobiol. 1988;163(1-3):202-207.

8. Walters MS, Shaykhiev R, Crystal RG. Cilia Dysfunction in Lung Disease. Anпи Rev Physio. 2015;77:379-406.
9. Tilley AE, Walters MS, Shaykhiev R, et al. Cilia Dysfunction in Lung Disease. Annu Rev Physiol. 2015;77:379-406.

10. Bachert C, P Gevaert, P van Cauwenberge. Nasal Polyps and Rhinosinusitis. In: NF Adkinson, et al. (Eds.), Middleton's Allergy:Principles and Practice edited. (7th edn), Mosby Elsevier, USA. 2008.

11. Stanley PJ, Wilson R, Greenstone MA, et al. Effect of cigarette smoking on nasal mucociliary clearance and ciliary beat frequency. Thorax. 1986;41(7):519-523.

12. Bossi R, Piatti G, Roma E, et al. Effects of long-term nasal continuous positive airway pressure therapy on morphology, function, and mucociliary clearance of nasal epithelium in patients with obstructive sleep apnea syndrome. Laryngoscope. 2004;114(8):1431-144.

13. Grossan M. Enhancing the Mucociliary System. Advance for Respiratory Care Practitioners. 1995;8:12-13.

14. Fontanari P, Burnet H, Zattara-Hartmann MC, et al. Changes in Airway Resistance Induced by Nasal Inhalation of Cold Dry, Dry, or Moist Air in Normal Individuals. J Appl Physiol. 1996; 81(4):1739-1743.

15. Boek WM, Keleş N, Graamans K, et al. Physiologic and Hypertonic Saline Solutions Impair Ciliary Activity In Vitro. Laryngoscope. 1999;109(3):396-399.

16. Laberko EL, Bogomil'sky MR, Soldatsky YL, et al. The influence of an isotonic solution containing benzalkonium chloride and a hypertonic seawater solution on the function of ciliary epithelium from the nasal cavity in vitro. Vestn Otorinolaringol. 2016;81(2):49-52.

17. Sisson JH, Pavlik JA, Wyatt TA. Alcohol stimulates ciliary motility of isolated airway axonemes through a nitric oxide, cyclase, and cyclic nucleotide-dependent kinase mechanism. Alcohol Clin Exp Res. 2009;33(4):610-616.

18. Eighteen Grossan M. Nasal Function: Office Measurement of Nasal Mucociliary Clearance. In Otolaryngologyedited by English. Willians Wilkins, Philadelphia, USA. 1994. 